\title{
Targeting inflammation to reduce seizure severity in an experimental model of eclampsia
}

\author{
Frank T. Spradley ${ }^{1}$
}

Received: 2 August 2019 / Revised: 6 August 2019 / Accepted: 6 August 2019 / Published online: 14 January 2020

(C) The Japanese Society of Hypertension 2020

Eclampsia, which refers to new-onset seizures in pregnant women without a history of seizure disorder, is associated with high maternal and fetal morbidity and mortality. Most commonly, eclampsia occurs as a progression from preeclampsia (PE), a disorder of new-onset hypertension and cardiovascular, renal, visual, and brain disturbances in the latter half of pregnancy. The incidence rate of PE ranges from 2 to $5 \%$ in developed countries and up to $18 \%$ in parts of Africa [1]. If PE is severe enough and left untreated, neurological problems can result. The brain can be affected, resulting in abnormal cerebral vascular function, blood brain barrier leakage, and edema that can cause the convulsive seizures that define eclampsia; this can ultimately result in death from hemorrhagic stroke. The rate of eclampsia is $\sim 0.05 \%$, but can reach $0.8 \%$ in developing countries, and eclampsia contributes to $7 \%$ of maternal deaths and $18 \%$ of fetal deaths [2]. Therefore, research on this area of maternal health is important for identifying mechanistic targets that translate to cost-effective treatments for PE and eclampsia.

Currently, magnesium sulfate $\left(\mathrm{MgSO}_{4}\right)$ is the primary treatment and it is administered prophylactically to women with severe PE who are at risk of developing eclampsia. While $\mathrm{MgSO}_{4}$ is effective in preventing seizures, it is not as effective in reducing hypertension or proteinuria in $\mathrm{PE}$ patients. Thus, finding a therapeutic agent that improves multiple PE symptoms is necessary. This issue of Hypertension Research includes a study conducted by Huang et al. entitled 'Cyclosporin A ameliorates eclampsia seizure through reducing systemic inflammation in an eclampsialike rat model'. By utilizing an experimental animal model, this study provides some important information about the

Frank T. Spradley

fspradley@gmail.com

1 Department of Surgery, The University of Mississippi Medical Center, Jackson, MS, USA mechanistic basis of the onset of eclamptic seizures. The purpose of this editorial is to summarize this original manuscript and to review the strengths and limitations of this study in which the authors examined the mechanisms that mediate the dangerous progression of PE to eclamptic seizures.

PE and eclampsia develop suddenly in humans, but there are no experimental research models to date that spontaneously develop these maternal disorders. Thus, investigators rely upon pharmacological and/or surgical models. This study by Huang and colleagues used a model of PE generated by injecting an ultralow dose of lipopolysaccharide (LPS) endotoxin on gestational day 14 followed by the I.P. injection of pentylenetetrazol (PTZ, a GABA receptor antagonist) at the end of gestation on day 20 to induce seizures. This paradigm was based on previous studies by the investigators in which LPS-induced preeclamptic rats, compared with normal pregnant rats, exhibited increased susceptibility to PTZ-induced seizures. In addition, the authors showed in earlier studies that the LPS model of PE exhibits the shortest latency to develop PTZ-induced seizures, the longest seizure duration, and the highest recorded EEG amplitudes along with neuronal loss in the hippocampus [3]. Neuronal hyperactivity in this brain region is linked to the onset of seizures [4]. While the observed functional and morphological changes are associated with central and systemic inflammation, the previous studies did not examine whether the increased susceptibility to seizures is mediated by pro-inflammatory pathways. The novelty of the current study is that the authors examined the causative pathways, whereby PE increases the risk for eclampsia.

The present study confirmed that LPS-induced preeclamptic rats have increased blood pressure (as determined by tail cuff plethysmography), albuminuria, seizure severity, and systemic inflammation by day 19/20 (Fig. 1). The pro-inflammatory markers included elevations in the levels of interleukin (IL)- $1 \beta$, tumor necrosis factor (TNF)- $\alpha$, and IL-17 in the circulation. To begin examining the role of pro-inflammatory pathways in mediating increased 


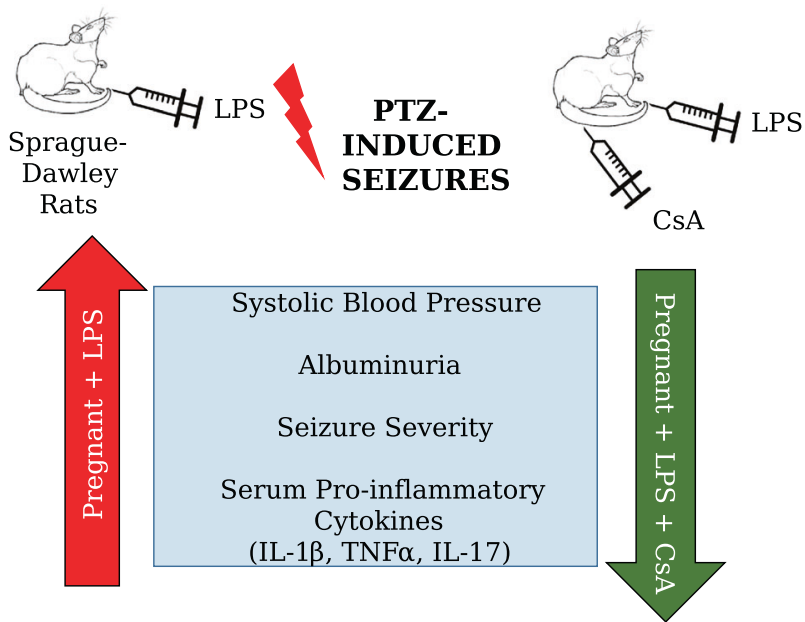

Fig. 1 Summary of findings from the Huang et al. study. LPS elicited preeclampsia-like symptoms and PTZ-induced seizure susceptibility in pregnant rats, which were attenuated by the immunosuppressant CsA

PTZ-induced seizure susceptibility in PE, rats were treated I.V. with the immunosuppressive agent cyclosporin A (CsA, a calcineurin inhibitor) from gestational day 16-18 according to the following three experimental groups: the normal pregnant $(\mathrm{P})$ group, $\mathrm{PE}$ group, and $\mathrm{PE}+\mathrm{CsA}$ group. By day 16, the PE rats developed hypertension and albuminuria. The initiation of CsA administration on day 16 lowered blood pressure and albuminuria in the PE rats to similar levels as those in the P rats when measured on day 19. The authors then used the three following groups to examine whether the increased susceptibility to seizures in the PE + PTZ rats was mediated by inflammation: the $\mathrm{P}+$ PTZ group, PE + PTZ group, and PE + PTZ + CsA group. Again, CsA administration was started on gestational day 16, PTZ was injected I.P. on day 20 and then the rats were immediately monitored for seizure severity. The elevated seizure scores and shortened latency to seizure in the PE + PTZ group compared with the P + PTZ group were significantly improved by CsA. CsA also abolished the elevation of IL-1 $\beta$, TNF- $\alpha$, and IL-17 levels in the PE model, which is not too surprising because of its known antiinflammatory action. This implicates circulating immune factors as contributors to exaggerated seizure susceptibility in PE.

It is important to note that both the levels of inflammatory cytokines and blood pressure were significantly reduced by CsA treatment in this study; therefore, at this time point, the relative contributions of elevated blood pressure and inflammation to these outcomes are unclear. It is likely that hypertension and circulating pro-inflammatory factors work together to increase the risk for the onset of eclamptic seizures. In women with PE and eclampsia, blood pressure values are significantly correlated with brain edema [5]. It has been shown in normal late-term pregnant rats that acute and dramatic increases in blood pressure to $\sim 180 \mathrm{mmHg}$ result in autoregulatory breakthrough, leading to edema formation, as measured by whole brain water content [6]. In regards to PE, Warrington et al. used a surgical model of PE, specifically the reduced uterine perfusion pressure (RUPP) model of placental ischemia, which presents with hypertension, a vast pro-inflammatory circulating milieu not limited to elevated TNF- $\alpha$ and IL-17 levels, reduced autoregulatory breakthrough threshold and increased cerebral blood flow, blood brain barrier permeability, and edema in the anterior cerebrum [7]. It was shown that RUPP rats have increased PTZ-induced seizure susceptibility and levels of cerebrospinal fluid cytokines, including IL-17 [7]. When IL-17 was infused into normal pregnant rats, blood brain barrier permeability and cortical brain water content increased [8]. However, whether cytokine blockade attenuates pressure-driven autoregulatory breakthrough and edema to mediate seizure susceptibility in these preeclamptic models (RUPP, LPS, cytokine infusion) has not been examined.

The aquaporin (AQP) water channels are potential molecular mechanisms downstream of the systemic inflammation in PE that have been shown to regulate brain water content, neuronal excitability, and seizures [9]. Huang et al. previously described that PTZ administration from gestational day 16-18 in their LPS-induced PE model results in neuronal death likely due to hyperexcitability resulting from greater AQP-4 and AQP-9 levels in the hippocampus [10]. Inflammation in the hippocampus promotes neuronal excitability, as demonstrated in acute experiments using rat hippocampal sections in which LPS was shown to directly promote neuronal hyperexcitability via TLR4 [11]. That study did not examine AQPs; however, in other studies, LPS was shown to induce AQP-4 in glial cells [12], and others have shown that AQP-4 itself is capable of eliciting hippocampal inflammation [13]. Future studies should determine whether LPS alone or PE-related systemic cytokines regulate AQPs to potentiate neuroinflammation, neuronal hyperexcitability, and the development of eclamptic seizures in an LPS-induced PE model.

Experimental animal studies, such as the present study, have suggested that PE-related inflammation is a target for preventing eclamptic seizures. In addition to inflammatory factors, PE is accompanied by elevated levels of other circulating factors, including the antiangiogenic molecule soluble fms-like tyrosine kinase-1 (sFlt-1); this factor targets vascular endothelial growth factor and placental growth factor, and reduces their bioavailability and vasoprotective actions, leading to increased blood pressure [14]. Although the objective of the Huang study was to utilize LPS to promote inflammation during pregnancy, in vitro studies have shown that LPS induces sFlt-1 production in human monocytes [15]. Others have shown that $\mathrm{T}$ lymphocytes are 
a source of sFlt-1 in response to placental ischemia in the RUPP model [16]. It is unknown how the antiangiogenic mechanisms of hypertension and vascular dysfunction were impacted by CsA-mediated immunosuppression in this study.

The Huang study involved treating PE rats with the immunosuppressant CsA for several days prior to the induction of PTZ-induced seizures. Although this afforded important mechanistic insight into how PE sensitizes rats to the onset of seizures, this treatment timing may not be clinically possible. Clinically, eclampsia can occur without warning signs and requires immediate treatment after the onset of high blood pressure and seizures [17]. At this point, experiments designed to examine whether immunosuppressant treatment can reverse eclamptic seizures and prevent neuronal damage in experimental models of PE have not yet been performed.

A treatment that is currently clinically approved for seizure prophylaxis in $\mathrm{PE}$ and for preventing the recurrence of seizures in eclampsia is $\mathrm{MgSO}_{4}$. In a previous study by the research team of the Huang study, $\mathrm{MgSO}_{4}$ was administered to LPS-induced PE models for several days before PTZ-induced seizures were examined. $\mathrm{MgSO}_{4}$ protected against astrocyte and microglial activation, promoted neuronal survival in the hippocampus, and prevented rises in the levels of cytokines in the cerebrospinal fluid, AQP-4 protein expression in the cortex, brain edema, and seizure susceptibility [3, 10, 18, 19]. However, they did not examine whether $\mathrm{MgSO}_{4}$ has an impact on other symptoms of $\mathrm{PE}$, such as maternal blood pressure. $\mathrm{MgSO}_{4}$ might not significantly impact the pathogenesis of hypertension in PE, as its administration did not affect the development of placental ischemia-induced hypertension in the RUPP rat model [20]. However, the present study found that CsA reduced maternal blood pressure and albuminuria and attenuated seizure susceptibility during PE. These basic research findings might be translatable to humans. The American College of Obstetrics and Gynecology's opinion is that CsA is safe in pregnancy [21]. Future studies should examine whether the immunosuppressants provided in the clinic are suitable for prophylaxis for blood pressure regulation in PE and eclampsia.

Acknowledgements This work was supported by funding from NHLBI via HL130577.

\section{Compliance with ethical standards}

Conflict of interest The author declares that they have no conflict of interest.

Publisher's note Springer Nature remains neutral with regard to jurisdictional claims in published maps and institutional affiliations.

\section{References}

1. Ronsmans C, Graham WJ, Lancet Maternal Survival Series Steering GroupMaternal mortality: who, when, where, and why. Lancet. 2006;368:1189-200.

2. Vousden N, Lawley E, Seed PT, Gidiri MF, Goudar S, Sandall J, et al. Incidence of eclampsia and related complications across 10 low- and middle-resource geographical regions: Secondary analysis of a cluster randomised controlled trial. PLoS Med. 2019;16: e1002775.

3. Liu L, Han X, Huang Q, Zhu X, Yang J, Liu H. Increased neuronal seizure activity correlates with excessive systemic inflammation in a rat model of severe preeclampsia. Hypertens Res. 2016;39:701-8.

4. Nakahara S, Adachi M, Ito H, Matsumoto M, Tajinda K, van Erp TGM. Hippocampal pathophysiology: commonality shared by temporal lobe epilepsy and psychiatric disorders. Neurosci J. 2018;2018:4852359.

5. Xiaobo F, Yanling L, Dunjin C, Fang H, Jia C, Yuhua Z, et al. Effect of blood pressure on reversible posterior leukoencephalopathy syndrome in pre-eclampsia or eclampsia. Hypertens Res. 2018;41:112-7.

6. Euser AG, Cipolla MJ. Cerebral blood flow autoregulation and edema formation during pregnancy in anesthetized rats. Hypertension. 2007:49:334-40.

7. Warrington JP, Fan F, Murphy SR, Roman RJ, Drummond HA, Granger JP, et al. Placental ischemia in pregnant rats impairs cerebral blood flow autoregulation and increases blood-brain barrier permeability. Physiol Rep. 2014;2:e12134.

8. Warrington J, Duncan D, Drummond HA, Granger JP, Ryan MJ Interleukin-17 infusion promotes cerebral edema and increased blood brain barrier permeability in pregnant rats. Hypertension. 2017;70:AP263.

9. Filippidis AS, Conroy TJ, Maragkos GA, Holsapple JW, Davies KG. Intraocular silicone oil migration into the ventricles resembling intraventricular hemorrhage: case report and review of the literature. World Neurosurg. 2017;102:695.e7-e10.

10. Han X, Huang Q, Liu L, Sha X, Hu B, Liu H. Changes in the expression of AQP4 and AQP9 in the hippocampus following eclampsia-like seizure. Int J Mol Sci. 2018;19:E300.

11. Isbrandt D. A mechanistic link between glia and neuronal excitability in acute neuroinflammation. J Physiol. 2017;595:603-4.

12. Sugimoto N, Leu H, Inoue N, Shimizu M, Toma T, Kuroda M, et al. The critical role of lipopolysaccharide in the upregulation of aquaporin 4 in glial cells treated with Shiga toxin. J Biomed Sci. 2015;22:78.

13. Yu H, Qi GL, Wang J, Chen L, Deng Z, Zhao YS, et al. Aquaporin 4 inhibition decreased synthesis of cytokines by acetazolamide in the hippocampus of rats with pentrazol-induced chronic epilepsy. Genet Mol Res. 2016;15:gmr9012.

14. Maynard SE, Min JY, Merchan J, Lim KH, Li J, Mondal S, et al. Excess placental soluble fms-like tyrosine kinase 1 (sFlt1) may contribute to endothelial dysfunction, hypertension, and proteinuria in preeclampsia. J Clin Invest. 2003;111:649-58.

15. Major HD, Campbell RA, Silver RM, Branch DW, Weyrich AS. Synthesis of sFlt-1 by platelet-monocyte aggregates contributes to the pathogenesis of preeclampsia. Am J Obstet Gynecol. 2014;210:547.e1-e7.

16. Wallace K, Richards S, Dhillon P, Weimer A, Edholm ES, Bengten E, et al. CD4 + T-helper cells stimulated in response to placental ischemia mediate hypertension during pregnancy. Hypertension. 2011;57:949-55.

17. Patwardhan VB. Eclampsia. J Indian Med Assoc. 1995;93: 58-9. 48 
18. Li X, Han X, Yang J, Bao J, Di X, Zhang G, et al. Magnesium sulfate provides neuroprotection in eclampsia-like seizure model by ameliorating neuroinflammation and brain edema. Mol Neurobiol. 2017;54:7938-48.

19. Li X, Liu H, Yang Y. Magnesium sulfate attenuates brain edema by lowering AQP4 expression and inhibits glia-mediated neuroinflammation in a rodent model of eclampsia. Behav Brain Res. 2019;364:403-12.
20. Zhang LW, Warrington JP. Magnesium sulfate prevents placental ischemia-induced increases in brain water content and cerebrospinal fluid cytokines in pregnant rats. Front Neurosci. 2016;10:561.

21. Committee on Obstetric Practice, Society for Maternal-Fetal Medicine. ACOG Committee opinion no. 776: immune modulating therapies in pregnancy and lactation. Obstet Gynecol. 2019;133:e287-e295. 